\title{
Design and Development of an Improved Adaptive Median Filtering Method for Impulse Noise Detection
}

\author{
Mamta Juneja, Parvinder Singh Sandhu
}

\begin{abstract}
An Impulse noise detection and removal with adaptive filtering approach is proposed to restore images corrupted by salt \& pepper noise. The proposed algorithm works well for suppressing impulse noise with noise density from 5 to $60 \%$ while preserving image details. The difference of current central pixel with median of local neighborhood pixels is used to classify the central pixel as noisy or noise-free. The noise is attenuated by estimating the values of the noisy pixels with a switching based median filter applied exclusively to those neighborhood pixels not labeled as noisy. The size of filtering window is adaptive in nature, and it depends on the number of noise-free pixels in current filtering window. Simulation results indicate that this filter is better able to preserve 2-D edge structures of the image and delivers better performance with less computational complexity as compared to other denoising algorithms existing in literature.
\end{abstract}

Index Terms-Image denoising, impulse noise, nonlinear filter, switching median filter.

\section{INTRODUCTION}

DIGITAL images could be contaminated by impulse noise during image acquisition or transmission. The intensity of impulse noise has the tendency of being either relatively high or relatively low. Thus, it could severely degrade the image quality and cause great loss of information details. So it is important to eliminate noise in the images before some subsequent processing, such as edge detection, image segmentation and object recognition. Two types of impulse noise can be modeled: i) Fixed valued impulse noise (salt \& pepper) and ii) Random valued impulse noise.

Various filtering techniques have been proposed for removing impulse noise in the past, and it is well-known that linear filters could produce serious image blurring. As a result, nonlinear filters have been widely exploited due to their much improved filtering performance, in terms of impulse noise attenuation and edge/details preservation. One of the most popular and robust nonlinear filters is the standard median (SM) filter [1], which exploits the rank-order information of pixel intensities within a filtering window and replaces the center pixel with the median value.

Manuscript received June 24, 2009. Er. Mamta Juneja is working with Department of Computer Science and Engineering as Assistant Professor in Rayat and Bahra Institute of Engineering and Bio-Technology, Sahauran, Distt. Mohali (Punjab)-140104 INDIA. er_mamta@yahoo.com,098786-77624

Dr. Parvinder S. Sandhu is working with Computer Science and Engineering Department as Professor and Head in Rayat and Bahra Institute of Engineering \& Bio-Technology, Sahauran, Distt. Mohali (Punjab)-140104 INDIA
Due to its effectiveness in noise suppression and simplicity in implementation, various modifications of the SM filter have been introduced, such as the weighted median (WM)[2] filter and the center weighted median (CWM) [3] filter.

Conventional median filtering approaches apply the median operation to each pixel unconditionally, that is, without considering whether it is uncorrupted or corrupted. As a result, even the uncorrupted pixels are filtered, and this causes image quality degradation. An intuitive solution to overcome this problem is to implement an impulse-noise detection mechanism prior to filtering; hence, only those pixels identified as "corrupted" would undergo the filtering process, while those identified as "uncorrupted" would remain intact. By incorporating such noise detection mechanism or "intelligence" into the median filtering framework, the so-called switching median filters [4]-[10] had shown significant performance improvement.

The most popular approaches for dealing with such noise have been based on median filtering and/or on the rich class of order statistic filters that have emerged from the study of median filters [11]. Recently, variations on the median filtering scheme have been shown, under various specific signal/noise models, to deliver improved performance relative to the corresponding traditional methods. Examples include the minimum-maximum exclusive mean filter (MMEM) [12], Florencio's [5], conditional median filtering (CMF) [16], Signal-dependent rank-order mean (SDROM) filter [17]. These filters have all demonstrated excellent performance, but at the price of significant computational complexity. The main drawback that characterizes all approaches of this type is that they involve computational overhead to determine one or more of the local order statistics.

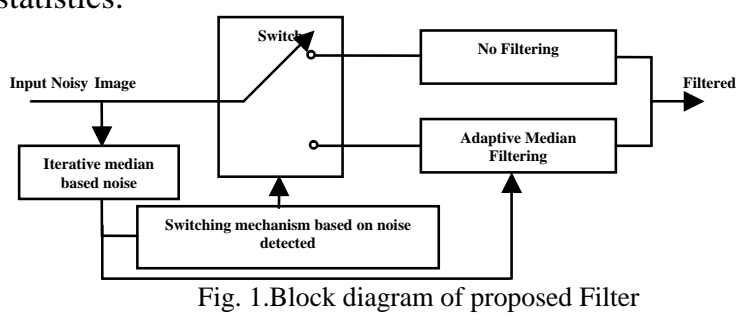

To solve this problem, a switching based adaptive median filtering scheme is proposed in this paper. The new scheme is illustrated in Fig.1. It exhibits improved performance in removing impulse noise while preserving fine details of the 2-D image structure.

The rest of the paper is organized as follows: Impulse noise detection is described in Section II. Section III introduces adaptive filtering scheme. Experimental result is 
shown in Section IV. Finally the paper is concluded in Section V.

\section{IMPULSE NOISE DETECTION}

The impulse detection is usually based on the following two assumptions: 1) a noise-free image consists of locally smoothly varying areas separated by edges and 2) a noisy pixel has tendency of very high or very low gray value compare to its neighbors. Two image sequences are generated during the impulse detection procedure. The first is a sequence of gray scale images, $\left\{x_{(i, j)}^{(0)}, x_{(i, j)}^{(1)}, x_{(i, j)}^{(2)}, \ldots x_{(i, j)}^{(n)}\right\}$ where the initial image $x_{(i, j)}^{(0)}$ is noisy image itself,$(i, j)$ is position of pixel in image, it can be $1 \leq i \leq \mathrm{M}, 1 \leq j \leq \mathrm{N}$ where $\mathrm{M}$ and $\mathrm{N}$ are the number of the pixel in horizontal and vertical direction respectively, and $x_{(i, j)}^{(n)}$ is image after $n^{\text {th }}$ iteration. The second is a binary flag image sequence, $\left\{f_{(i, j)}^{(0)}, f_{(i, j)}^{(1)}, f_{(i, j)}^{(2)}, \ldots f_{(i, j)}^{(n)}\right\}$ where the binary flag $f_{(i, j)}^{(n)}$ is used to indicate whether the pixel at $(i, j)$ in noisy image detected as noisy or noise-free after $n^{\text {th }}$ iteration. If $f_{(i, j)}^{(n)}=0$ means pixel at $(i, j)$ has been found as noise-free after $n^{\text {th }}$ iteration and if $f_{(i, j)}^{(n)}=1$ means pixel at $(i, j)$ has been found as noisy after $n^{\text {th }}$ iteration. Before the first iteration, we assume that all the image pixels are good, i.e. $f_{(i, j)}^{(0)}=0$ for all $(i, j)$.

Steps for noise detection:

1. Lets take a $(2 \mathrm{~W}+1) \times(2 \mathrm{~W}+1)$ window around $x_{(i, j)}^{(n-1)}$ means

$$
x_{(i+k, j+l)}^{(n-1)} \text { where }-\mathrm{W} \leq k \leq \mathrm{W},-\mathrm{W} \leq l \leq \mathrm{W}
$$

and $\mathrm{W} \geq 1$.

2. Find Median value of this window $m_{(i, j)}^{(n-1)}$

$$
m_{(i, j)}^{(n-1)}=\operatorname{median}\left(x_{(i+k, j+l)}^{(n-1)}\right)
$$

3. Find absolute difference between $x_{(i, j)}^{(n-1)}$ and

$$
\begin{aligned}
& m_{(i, j)}^{(n-1)} \text {, and assign } \\
& f_{(i, j)}^{(n)}= \begin{cases}f_{(i, j)}^{(n-1)}, & \text { if }\left|x_{(i, j)}^{(n-1)}-m_{(i, j)}^{(n-1)}\right|<T \\
1, & \text { otherwise }\end{cases}
\end{aligned}
$$

(2)

Where $T$ is predefined threshold value. 1 indicates pixel detected as noisy after $n^{\text {th }}$ iteration.

4. If $(i, j)^{\text {th }}$ is detected as noisy then the value of $x_{(i, j)}^{(n)}$ will be modified as

$$
x_{(i, j)}^{(n)}= \begin{cases}m_{(i, j)}^{(n-1)}, & \text { if } f_{(i, j)}^{(n)} \neq f_{(i, j)}^{(n-1)} \\ x_{(i, j)}^{(n-1)}, & \text { if } f_{(i, j)}^{(n)}=f_{(i, j)}^{(n-1)}\end{cases}
$$

This all steps will repeat for $t$ times. This $t$ can be $2,3,4 \ldots$..After the $t^{\text {th }}$ iteration we have two images $x_{(i, j)}^{(t)}$ and $f_{(i, j)}^{(t)}$. But only $f_{(i, j)}^{(t)}$ binary flag image is required for noise filtering process. This median based noise detection had introduced by Sun and Neuvo [4] and it is modified by Wang and Zhang (PSM) [7] in progressive way. The difference between PSM [7] and our approach is, we have applied adaptive filtering approach for improving filtering performance of the filter. From the simulation result we can see our approach is giving better performance in term of PSNR and visual aspect.

\section{ADAPTIVE NOISE FILTERING}

From the last section we got binary flag image $f_{(i, j)}^{(t)}$ which elements give information about whether the pixel is corrupted or not corrupted at location $(i, j)$ in noisy image $x_{(i, j)}^{(0)}$.If $(i, j)^{\text {th }}$ pixel has detected as a noise then it will go through median filtering process other wise it will remain same. This is called Switching based median filter. Here the size of filtering window is adaptive in nature and its size is depend on the number of pixels which are noise free in current filtering window. The maximum window size shouldn't be more than $7 \times 7$ to reduce blurring effect. Steps are given below for Adaptive Switching Filtering:

1. Start with $(3 \times 3)$ filtering window form $x_{(i, j)}^{(0)}$ and corresponding $(3 \times 3)$ window from binary flag image $f_{(i, j)}^{(t)}$.

2. Find out how many pixels are detected as noise-free in current filtering window from corresponding binary flag window.

3. Iteratively extends window size outward by one pixel in all the four sides of the window, if the number of uncorrupted pixels is less than half of the total number of pixels (denoted by $S_{\text {in }}=1 / 2[3 \times 3]$ ) within the filtering window. These all above three steps should be repeat again if condition are not satisfy.

4. So since the current pixel has been marked noisy, it will not participate in filtering process. Only the pixels that are classified as noise free in filtering window will participate in median filtering process. This will, in turn, yield a better filtering result with less distortion.

\section{SIMULATION RESULTS}

Intensive simulations were carried out using several monochrome images, from which "Lena," "Peppers," and "Bridge" are chosen for demonstrations. The performance evaluation of the filtering operation is quantified by the PSNR (peak signal to noise ratio) calculated using formula:

$$
\text { PSNR }=10 \log _{10}\left(\frac{255^{2}}{\mathrm{MSE}}\right)
$$

Where

$$
\mathrm{MSE}=\frac{1}{\mathrm{MN}} \sum_{i=1}^{M} \sum_{j=1}^{N}[I(i, j)-\hat{I}(i, j)]^{2}
$$

Where $\mathrm{M}$ and $\mathrm{N}$ are the total number of pixels in the horizontal and the vertical dimensions of the image. $I$ and $\hat{I}$ denote the original and filtered image, respectively. 
Table 1. PSNR Performance of Different Algorithms for Lena image corrupted with salt and pepper noise

\begin{tabular}{|c|c|c|c|}
\hline \multirow{2}{*}{ Algorithm } & \multicolumn{3}{|c|}{ Lena Corrupted with Noise density } \\
\hline & $10 \%$ & $20 \%$ & $30 \%$ \\
\hline $\operatorname{MF}(3 \times 3)$ & $31.19 \mathrm{~dB}$ & $28.48 \mathrm{~dB}$ & $25.45 \mathrm{~dB}$ \\
\hline $\operatorname{MF}(5 \times 5)$ & $29.45 \mathrm{~dB}$ & $28.91 \mathrm{~dB}$ & $28.43 \mathrm{~dB}$ \\
\hline MMEM [13] & $30.28 \mathrm{~dB}$ & $29.63 \mathrm{~dB}$ & $29.05 \mathrm{~dB}$ \\
\hline Florencio's[5] & $33.69 \mathrm{~dB}$ & $32.20 \mathrm{~dB}$ & $30.95 \mathrm{~dB}$ \\
\hline PMCWF [14] & $35.70 \mathrm{~dB}$ & $32.95 \mathrm{~dB}$ & $31.86 \mathrm{~dB}$ \\
\hline $\operatorname{AMF}(3 \times 3)[15]$ & $33.79 \mathrm{~dB}$ & $30.65 \mathrm{~dB}$ & $26.26 \mathrm{~dB}$ \\
\hline $\operatorname{AMF}(5 \times 5)[15]$ & $30.11 \mathrm{~dB}$ & $28.72 \mathrm{~dB}$ & $27.84 \mathrm{~dB}$ \\
\hline $\mathrm{CMF}(3 \times 3)[16]$ & $38.05 \mathrm{~dB}$ & $31.79 \mathrm{~dB}$ & $26.22 \mathrm{Db}$ \\
\hline $\operatorname{CMF}(5 \times 5)[16]$ & $36.32 \mathrm{~dB}$ & $33.52 \mathrm{~dB}$ & $30.33 \mathrm{~dB}$ \\
\hline SDROM [17] & $37.93 \mathrm{~dB}$ & $34.10 \mathrm{~dB}$ & $29.80 \mathrm{Db}$ \\
\hline Proposed & $42.14 \mathrm{~dB}$ & $38.66 \mathrm{~dB}$ & $35.75 \mathrm{Db}$ \\
\hline
\end{tabular}

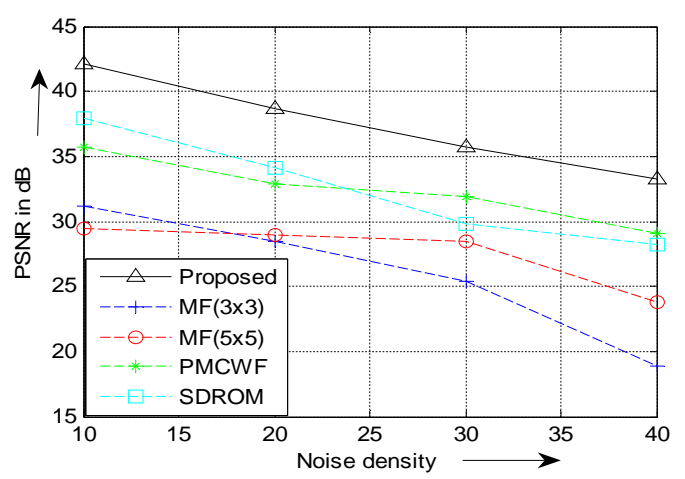

Fig.2. PSNR Plot for Lena image corrupted with different noise density

This proposed technique is compared with different standard methods that are standard median filters $\operatorname{MF}(3 \times 3)$, $\mathrm{MF}(5 \times 5)$, minimum-maximum exclusive mean $(\mathrm{MMEM})$ filter[12] , Florencio's[5], conditional median filtering (CMF)[16], signal-dependent rank-order mean (SDROM) filter [17],progressive switching median filter [7]. The proposed method has been applied on Lena, Pepper and Bridge gray images of size $512 \times 512$ corrupted by fixed-value impulse noise with different densities ranges from $5 \%$ to $60 \%$.For simulation we have taken $\mathrm{T}=40$, and $t=2$. Comparatively PSNR performance has been given with different noise density in Table. 1 for Lena image.

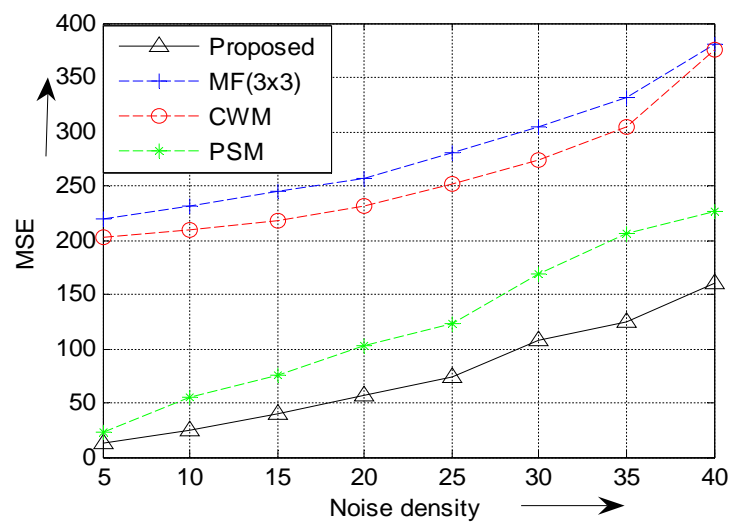

Fig.3. MSE Plot for Bridge image corrupted with different noise density

This PSNR performance is also plotted in fig.2.In fig. 4 test images, noisy images and corresponding denoised images are shown. For comparison with PSM [7] we have given MSE (mean square error) plot in fig. 3 with different noise density for Bridge image. From all this simulation results we can say performance of the proposed method is better than other methods in terms of PSNR and visual aspect.

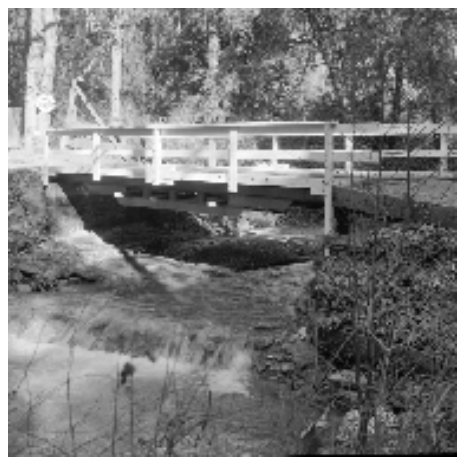

(a)

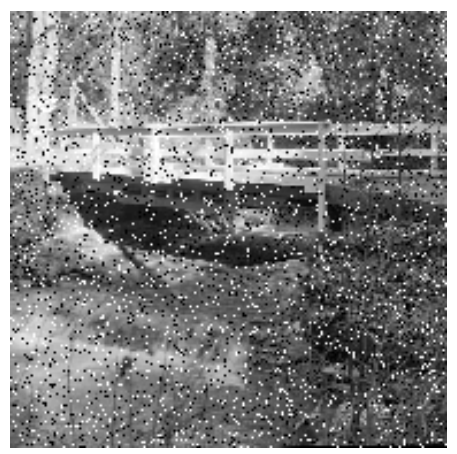

(b)

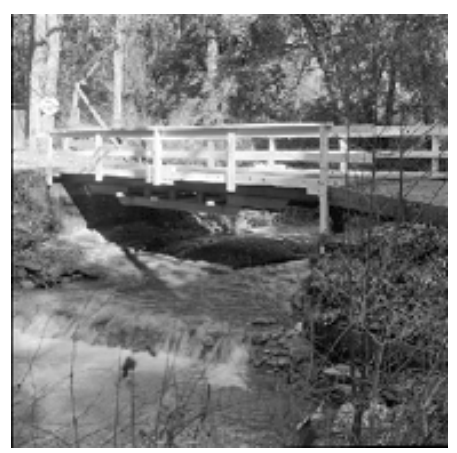

(c)

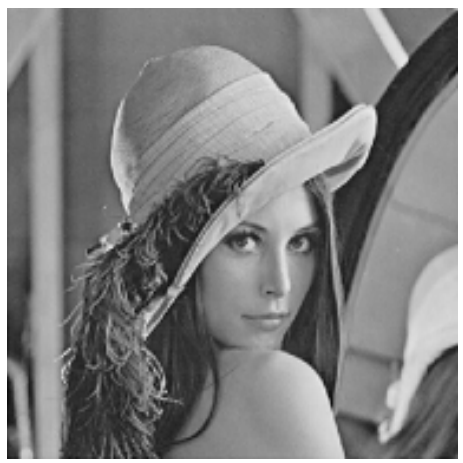

(d) 


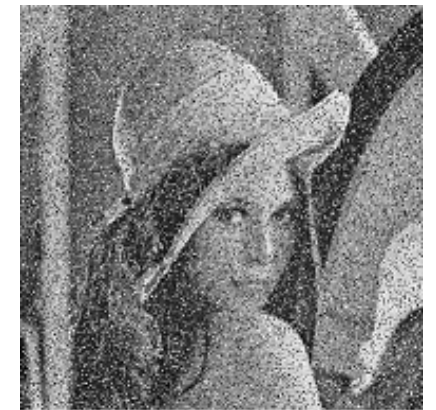

(e)

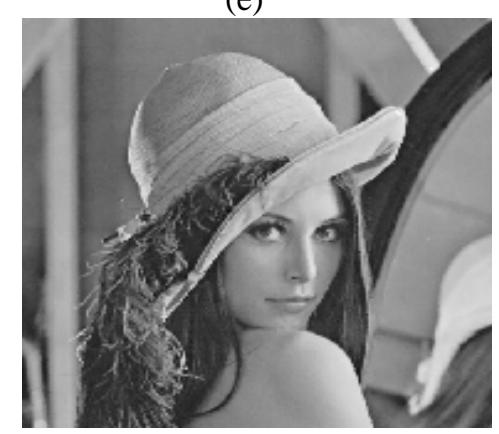

(f)

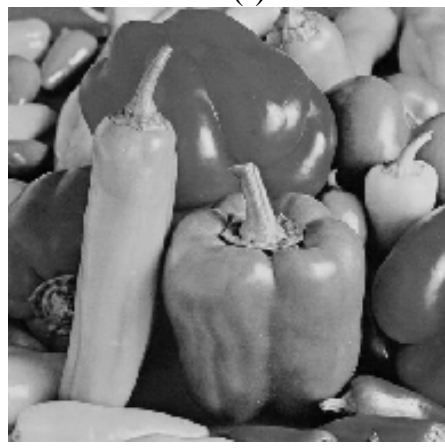

(g)

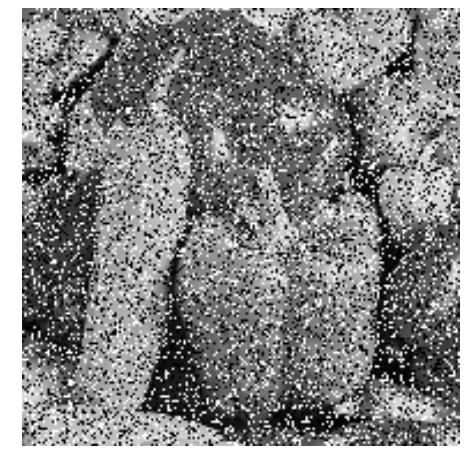

(h)

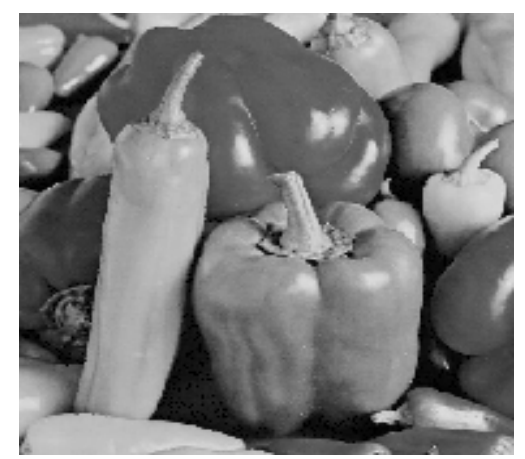

(i)

Fig.4. The original image (a)Bridge, (d)Lena, (g)Pepper and corresponding noisy image corrupted by $10 \%, 20 \%, 30 \%$ fixed value impulse noise is (b),(e),(h) respectively and (c),(f),(i) are filtered image of (b),(e),(h) respectively.

\section{CONCLUSION}

To demonstrate the performance of the proposed method, extensive simulation experiments have been conducted on a variety of standard test images to compare our method with many other well known techniques. Experimental results indicate that the proposed method performs significantly better than many other existing techniques. The proposed method is simple and easy to implement.

\section{REFERENCES}

[1] I. Pitas and A. N. Venetsanopoulos, "Order statistics in digital image processing," Proc. IEEE, vol. 80, no. 12, pp. 1893-1921, Dec. 1992.

[2] D. R. K. Brownrigg, "The weighted median filter," Commun. ACM, vol. 27, no. 8, pp. 807-818, Aug. 1984.

[3] S.-J. Ko and Y. H. Lee, "Center weighted median filters and their applications to image enhancement," IEEE Trans. Circuits Syst., vol. 38, no. 9, pp. 984-993, Sep. 1991.

[4] T. Sun and Y. Neuvo, "Detail-preserving median based filters in image processing," Pattern Recognit. Lett., vol. 15, no. 4, pp. 341-347, Apr. 1994.

[5] D. A. Florencio and R. W. Schafer, "Decision-based median filter using local signal statistics," in Proc. SPIE Vis. Commun. Image Process., vol. 2308, Sep. 1994, pp. 268-275.

[6] T. Chen, K.-K. Ma, and L.-H. Chen, "Tri-state median filter for image denoising," IEEE Trans. Image Process., vol. 8, no. 12, pp. 1834-1838, Dec. 1999.

[7] Z. Wang and D. Zhang, "Progressive switching median filter for the removal of impulse noise from highly corrupted images," IEEE Trans. Circuits Syst. II, vol. 46, no. 1, pp. 78-80, Jan. 1999.

[8] H.-L. Eng and K.-K. Ma, "Noise adaptive soft-switching median filter," IEEE Trans. Image Process., vol. 10, no. 2, pp. 242-251, Feb. 2001.

[9] G. Pok, J.-C. Liu, and A. S. Nair, "Selective removal of impulse noise based on homogeneity level information," IEEE Trans. Image Processing, vol. 12, no. 1, pp. 85-92, Jan. 2003.

[10] H. Hwang and R. A. Haddad, "Adaptive median filters: New algorithms and results," IEEE Trans. Image Process., vol. 4, no. 4, pp. 499-502, Apr. 1995

[11] A. Bovik, Handbook of Image \& Video Processing, ${ }^{\text {st }}$ Ed. New York: Academic, 2000.

[12] W.-Y. Han and J.-C. Lin, "Minimum-maximum exclusive mean (MMEM) filter to remove impulse noise from highly corrupted images," Electron. Lett., vol. 33, no. 2, pp. 124-125, 1997.

[13] T. Chen and H. R. Wu, "Adaptive impulse detection using center-weighted median filters," IEEE Signal Processing Lett., vol. 8, pp. 1-3, Jan. 2001.

[14] J.-H. Wang, "Prescanned minmax center-weighted filters for image restoration," Proc. Inst. Elect. Eng., vol. 146, no. 2, pp. 101-107, 1999.

[15] A. Sawant, H. Zeman, D. Muratore, S. Samant, and F. DiBianka, "An adaptive median filter algorithm to remove impulse noise in X-ray and CT images and speckle in ultrasound images," Proc. SPIE, vol. 3661, pp. 1263-1274, Feb. 1999.

[16] T. Kasparis, N. S. Tzannes, and Q. Chen, "Detail-preserving adaptive conditional median filters," J. Electron. Imag., vol. 1, no. 14, pp. 358-364, 1992.

[17] E. Abreu, M. Lightstone, S. K. Mitra, and K. Arakawa, "A new efficient approach for the removal of impulse noise from highly corrupted images," IEEE Trans. Image Processing, vol. 5, pp. 1012-1025, June 1996. 